\title{
The design of laboratory experiments to produce collisionless shocks of cosmic relevance
}

\author{
R. P. Drake \\ University of Michigan, Ann Arbor, Michigan 48109
}

(Received 14 March 2000; accepted 7 August 2000)

\begin{abstract}
Naturally occurring shocks transport energy and accelerate particles throughout the cosmos. The problem of producing collisionless shocks in the laboratory that are of relevance to such cosmic shocks is considered. Such an experiment must meet a number of constraints, several of which can be expressed by algebraic scaling relations. The relations for magnetization, plasma beta, Alfvén Mach number, temperature, magnetic field, and collisionality are described here. Taken together, the limits imposed by these constraints upon possible experiments are specified. The growth of magnetohydrodynamic (MHD) turbulence and the degree of particle acceleration are examined, demonstrating that it is feasible to contemplate studies of such phenomena in the laboratory. Finally, some discussion of how an experiment might meet the other qualitative constraints, and of how a laser might be used to drive the shock, is also included. (C) 2000 American Institute of Physics. [S1070-664X(00)04111-2]
\end{abstract}

\section{INTRODUCTION}

The proposal, by Roald Sagdeev, in the late 1950's, that shock waves could exist without collisions ${ }^{1}$ was quite controversial for a number of years, until the first observation of such shock waves in outer space. ${ }^{2}$ It has since become quite clear, from studies by satellites and spacecraft and from astrophysical simulations, that collisionless shocks play an important role in the transport of energy and the evolution of particle distributions in extraterrestrial plasmas. Specifically, it seems likely that supernova shocks are the dominant source of galactic cosmic rays $^{3-6}$ and that heliospheric shocks are a major contributor to the low-energy cosmic-ray spectrum. ${ }^{7-10}$ In the present paper, we are concerned with the potential of laboratory experiments to contribute to our knowledge of collisionless shocks as they occur in nature. We refer to such shocks here as "cosmic shocks."

The first attempts at laboratory simulations of space phenomena were reviewed ${ }^{11}$ by Podgorny and Sagdeev in 1970. Subsequent events were reviewed ${ }^{12}$ by Kennel et al. in the mid 1980's. By that time laboratory experiments had completely died out as funding decreased for magnetic-pinch fusion. The use of pinches for such experiments, in addition, had some limitations from the standpoint of relevance to cosmic shocks. The experiments were often too brief to allow the shock-reflected ions to participate in the further evolution of the shock. ${ }^{13}$ In addition, experiments with theta pinches could not produce a high-beta plasma with magnetized ions in which the electrons were unaffected by material walls. Enthusiasm remained, however, that observations in nearby outer space could provide the data necessary to develop a complete understanding. Over time this has not proven to be feasible, because of the incomplete ability to diagnose or to reproduce the shock waves encountered by spacecraft. It is now clear that laboratory experiments, when they can be sufficiently informative, will have much to add.
Cosmic shocks occur in systems in which both the electrons and the ions are well magnetized. They also evolve over distances sufficient for particle acceleration to occur through diffusive interaction with the magnetohydrodynamic (MHD) turbulence near the shock. In this context, the goal of laboratory studies of collisionless shocks must be to produce such shocks under conditions that do lead to particle acceleration, to measure in detail the MHD turbulence that they produce, and to diagnose the evolution of the particle distributions. Such laboratory experiments must meet a number of constraints, discussed more thoroughly in the following. The summary is that the particles must be well magnetized but yet the plasma pressure must be large enough to affect the magnetic field. The Alfvén waves must not outrun the shock, yet there must be enough time and space for the MHD turbulence to develop and for particles to be accelerated. This is quite a challenge. Magnetized laboratory plasmas typically do not produce shocks. In contrast, the laser-produced plasmas that do produce shocks are typically unmagnetized. In the present paper, we will systematically examine what is required to produce and study collisionless shocks in the laboratory.

The plan of the paper is as follows. In Sec. II we will briefly review past laboratory work on collisionless shocks. In Sec. III we will discuss the constraints that an experiment must meet, and in Sec. IV we will show how to meet the constraints involving magnetization, beta, and Mach number. In Sec. V we will discuss the implications for temperature and beta. Collisions will be the subject of Sec. VI. The growth of MHD turbulence and the degree of particle acceleration are discussed in Sec. VII. In Sec. VIII we will discuss the production and isolation of the plasma and in Sec. IX we will discuss the production of a "piston" that can drive the sort of shocks we find to be feasible. Section $\mathrm{X}$ is a conclusion. 


\section{CONTEXT}

Here we undertake a brief review of past, laboratory collisionless-shock studies. There were a number of experiments in the late 1960's and early 1970's. Most of these experiments took advantage of theta pinch coils, often supported for magnetic fusion research, to drive a perpendicular shock radially inward. They have been reviewed ${ }^{14,15}$ by Biskamp and by Hintz. These experiments were focused on the scaling of the shock structure with the Alfvén Mach number, $M_{\mathrm{A}}$, because the MHD theory of shock waves requires that their structure must change above a critical value of $M_{\mathrm{A}}$. Such changes were detected, ${ }^{16}$ but the experiments had a very limited ability to examine the details of the physical system. For example, the speed of the available electronics prevented such experiments from diagnosing the Alfvénic turbulence and the evolution of the particle distributions. In other work, some evidence was reported of collisionless coupling between ionized background plasma and expanding, laser-produced plasma, as discussed ${ }^{17}$ by Cheung et al. and the references therein. This work did not extend, however, to studies of collisionless shocks.

Recent years have included some experiments in which lasers have been used to drive shock waves. These are reviewed in a recent paper ${ }^{18}$ and some of them are relevant here. Experiments ${ }^{19}$ using a $\mathrm{CO}_{2}$ laser at Los Alamos produced copious energetic electrons which may have prevented the formation of an unmagnetized, collisionless shock. Experiments using $100 \mathrm{~J}$ class lasers at the Naval Research Laboratory, ${ }^{20}$ Lawrence Livermore National Laboratory, ${ }^{21}$ and Los Alamos have studied the blast waves driven by the expansion of a laser-irradiated target into neutral gas. These experiments are relevant to explosions within atmospheres but not directly to collisionless shocks. They do produce some hydrodynamic effects, such as the Vishniac instability, ${ }^{22}$ that may also develop in collisionless systems. Certain experiments in Russia are most relevant here, ${ }^{23,24}$ because they did produce collisionless shocks. In these experiments, an expanding, laser-produced plasma drove a shock wave through a plasma produced by a theta pinch. Early during the expansion, both the plasma $\beta$ and the Alfvénic Mach number were large. However, these quantities decreased below unity during the experiments, which limits their relevance to cosmic shocks. The ability to understand details and to test models was also limited, because only global measurements of the expanding structures were reported. Nonetheless these Russian experiments provided a significant motivation for the analysis discussed herein.

In addition, there was one report ${ }^{25}$ of a "shock" produced when a flowing laser plasma encountered an obstacle. In this case, the shock thickness measured by schlieren photography was much less that the collisional mean free paths inferred by the authors. The present author notes that this paper does not discuss the role of scattered laser radiation, or of the electron flux from the laser plasma, in evolving plasma from the obstacle. It is unclear to him whether the structures observed might not be better described as colliding plasmas, ${ }^{26-28}$ in which ion-ion two-stream instabilities provide thermalization, rather than as a shock. In any event,

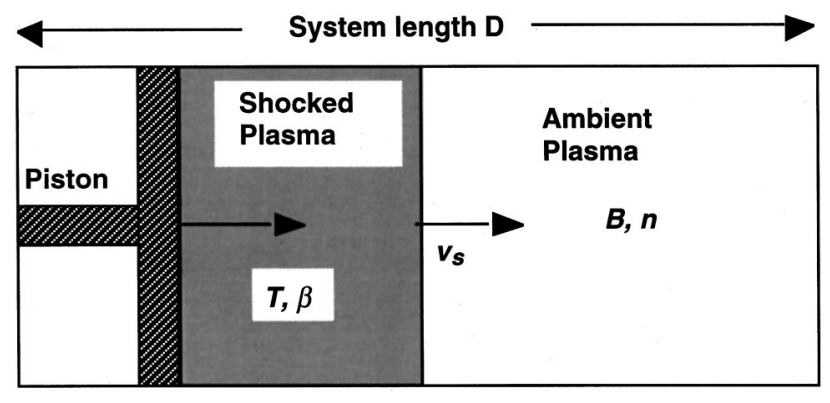

FIG. 1. Idealization of experiment, for analysis.

such "shocks" are not of cosmic relevance, which is our focus here.

In other related work, ${ }^{29,30}$ Ripin et al. studied the subAlfvénic expansion of a laser-produced plasma into a magnetic field. They explored the large-Larmor-radius variant of the Rayleigh Taylor instability that develops under such conditions.

\section{THE CONSTRAINTS}

The design of a collisionless shock experiment must satisfy a number of simultaneous constraints. We identify and discuss these in turn. For analysis of this system, we work with an idealization, shown in Fig. 1. A piston drives a shock through pre-existing plasma. The shock velocity is $v_{s}$, the post-shock temperature and beta are $T$ in $\mathrm{eV}$ and $\beta$, the preshock magnetic field is $\mathbf{B}$ in Gauss, the pre-shock density is $n$ in number per $\mathrm{cm}^{3}$, and the total distance over which the shock can be driven is $D$ in $\mathrm{cm}$.

We take in turn the following constraints that must be met. The experiment must have the following:

(a) A system size of many gyroradii (i.e., be highly magnetized)

(b) Plasma beta $(\beta)>1$, so that the plasma can readily affect the field

(c) Alfvén Mach number $>1$, so the upstream Aflvenic turbulence does not escape the shock

(d) "Collisionless" conditions, the details of which are discussed below

(e) A shock unaffected by material walls

(f) Plasma production that achieves well-defined initial conditions

(g) An adequate piston

(h) Enough time for MHD turbulence to grow at the shocks and for particle acceleration to occur

(i) Diagnosable parameters

A note regarding constraint $\mathrm{b}$ is that the $\beta$ is that of the shocked plasma. This should be sufficient to allow the shock and its precursors to produce MHD turbulence and to strongly affect the field $B$. It is not essential that the upstream, unperturbed plasma have significant $\beta$, although if achievable this would be desirable.

The primary focus of this paper will be upon constraints a through $\mathrm{d}$ and $\mathrm{h}$. These can be analyzed mathematically by means of scaling relations. Constraints e and $f$ are much 
more dependent upon the specific approach to an experiment. Only some general remarks will be offered about them. The treatment of the driving piston (constraint $\mathrm{g}$ ) is also specific to a given experiment. Some aspects of laser-driven pistons will, however, be discussed. Finally, the diagnosis of the experiment (constraint i) will also be left to specific experimental designs. One should note, though, that the spectacular advances in electronics during the 30 years since the prior laboratory experiments on this topic imply that the unstable dynamics could now be examined much more closely.

\section{MAGNETIZATION AND BETA}

The first problem faced by laboratory collisionless-shock experiments is that space and astrophysical plasmas are very magnetized and yet also have significant beta. The gyroradius of an ion is a tiny fraction of the size of structures of interest in a typical extraterrestrial system $\left(\sim 10^{-9}\right.$, for example, in supernova remnants $\left.{ }^{31}\right)$. Yet at the same time the magnetic field is weak, with $\beta$, the ratio of plasma pressure to magnetic field pressure, being of order 1 in space plasmas and often much larger in astrophysical ones. The problem in the lab is that a strongly magnetized plasma, having a small gyroradius compared to the experiment size, typically also has a small $\beta$. These two constraints are coupled as described next.

We define the magnetization, $a$, to be the ratio of system size to gyroradius, $a=D / r_{\mathrm{Li}}$. Here $r_{\mathrm{Li}}$ is the gyroradius of the shocked ions. We evaluate this as

$$
r_{\mathrm{Li}}=\frac{100 \sqrt{T}}{4 B} \frac{\sqrt{A}}{Z},
$$

in which the factor of 4 applies to perpendicular shocks, $A$ is the atomic mass of the ion, and $Z$ is its charge. This then implies that

$$
a=\frac{D B}{25 \sqrt{T}} \frac{Z}{\sqrt{A}} \text { or } a^{2}=\frac{D^{2}}{625} \frac{B^{2}}{T} \frac{Z^{2}}{A} .
$$

(In the case of parallel shocks, the field strength is not increased by the shock and the coefficients in these denominators are 100 and $10^{4}$, respectively.) For relevance to cosmic shocks, this parameter should be as large as possible. Assuming that the ions are magnetized, the electrons are extremely well magnetized, because their gyroradius is at most $1 / 40$ that of the ions.

One also desires the plasma pressure, $\beta$, to be as large as feasible. This is

$$
\beta=4 \times 10^{-11} \frac{(4 n) T}{(4 B)^{2}}=10^{-11} n \frac{T}{B^{2}},
$$

where the compressed density is $4 n$ and the compressed field is again $4 B$. (For parallel shocks, the coefficient $10^{-11}$ is replaced by $1.6 \times 10^{-10}$.) In writing Eq. (3), the electron temperature is assumed to be negligible, for reasons discussed below in Sec. VI. What is notable here is that $T / B^{2}$ appears again. Combining Eqs. (2) and (3), one finds

$$
\beta a^{2}=1.6 \times 10^{-14} n D^{2}\left(Z^{2} / A\right) .
$$

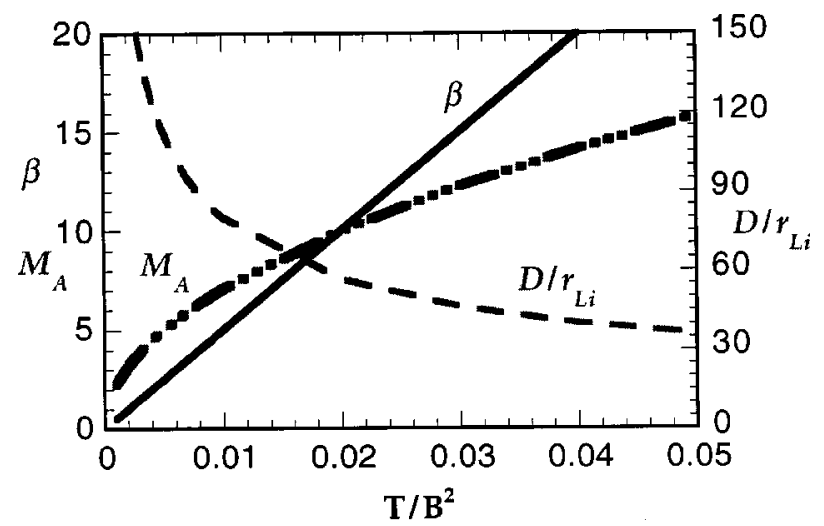

FIG. 2. Perpendicular shocks: magnetization vs $\beta$ tradeoff for $n D^{2}=2$ $\times 10^{18} \mathrm{~cm}^{-1} . M_{\mathrm{A}}$ is shown for $n=5 \times 10^{13} \mathrm{~cm}^{-3}$.

This expression is the same for both perpendicular and parallel shocks.

The Alfvén Mach number has a similar scaling to $\beta$, if we connect the shock velocity to $T$ by the standard relation, $T=(3 / 16) M_{i} v_{s}^{2}$, where $M_{i}$ is the mass of the ambient ions. Then

$$
M_{\mathrm{A}}=\frac{v_{s}}{v_{\mathrm{A}}}=10^{-5} \sqrt{n} \frac{\sqrt{T}}{B} .
$$

In addition, the duration of the experiment, in gyroperiods of the shocked ambient ions, is implied by the magnetization. In general, $\omega_{c i} \tau=\sqrt{3} a / 4$, because the ion thermal velocity, $v_{T} \equiv \sqrt{T / M_{i}}$, is related to the shock velocity by $v_{T}$ $=\sqrt{3} v_{s} / 4$, and the shock transit time is $\tau=D / v_{s}$ (in the approximation of constant $v_{s}$ ).

These equations define the fundamental tradeoffs for such an experiment, and show why system size, $D$, is very important. The density, $n$, will be limited by the requirement that the system be collisionless and by the limited energy available to sustain the shock and accelerate ambient ions. The tradeoff, for perpendicular shocks, described by Eqs. (1)-(5) is illustrated in Fig. 2, for $n D^{2}=2$ $\times 10^{18} \mathrm{~cm}^{-1} . M_{\mathrm{A}}$ is shown for $n=5 \times 10^{13}$ per $\mathrm{cm}^{3}$, corresponding to $D=200 \mathrm{~cm}$. (These values will turn out to be reasonable for an experiment once all the constraints have been taken into account.) One sees that achieving a magnetization of $\sim 100$ with significant $\beta$ and $M_{\mathrm{A}}$ is feasible for this case.

For parallel shocks, the tradeoff between magnetization and the other parameters is shown in Fig. 3. Here $\beta$ becomes larger but the magnetization becomes smaller, as is illustrated in Fig. 3 for the same experimental parameters. In this case, $\beta$ can be in the range of 5 to 10 while $a$ is in the range of 50 to 100 , with an $M_{\mathrm{A}}$ of about 2 .

In concluding this section, we note that the value of $n D^{2}$ used here could correspond equally well to other experiments, as is illustrated in Fig. 4. A corresponding theta pinch experiment, for example, would have $D \sim 20 \mathrm{~cm}$ and $n \sim 5$ $\times 10^{15}$ per $\mathrm{cm}^{3}$. (This is about 10 times the density obtained in the experiment ${ }^{16}$ of Phillips and Robson.) A corresponding dense laser-plasma experiment would have $D \sim 1 \mathrm{~mm}$ and $n \sim 2 \times 10^{20} / \mathrm{cm}^{3}$. Working at higher density does require 


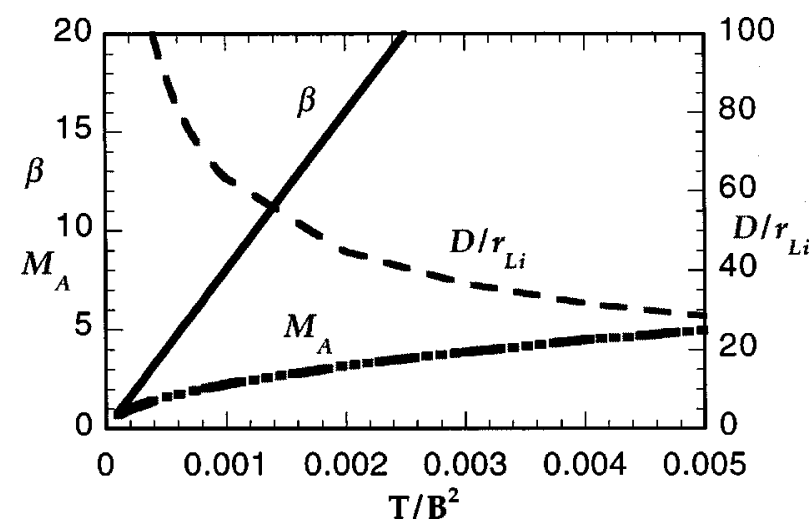

FIG. 3. Parallel shocks: magnetization vs $\beta$ tradeoff for $n D^{2}=2$ $\times 10^{18} \mathrm{~cm}^{-1} \cdot M_{\mathrm{A}}$ is shown for $n=5 \times 10^{13} \mathrm{~cm}^{-3}$.

less energy, as the total number of particles to be heated, N, scales as $N \propto\left(n D^{2}\right)^{3 / 2} / \sqrt{n}$. However, higher density produces more collisions. We will explore the limits due to collisions in Sec. VI, and will find the dense laser-plasma case to be unfeasible.

\section{TEMPERATURE AND MAGNETIC FIELD}

It is evident from the above discussion that $T$ and $B$ are also inherently coupled. It is also evident from Eqs. (1)-(5) that one pays a large penalty in magnetization by using any ion heavier than hydrogen, assuming that one cannot highly ionize such ions. We assume hydrogen ambient ions henceforth. For a specific shock geometry and for given values of any three of the quantities $D, n, \beta$, and $a$, the ratio of $T$ to $B^{2}$ is fixed by the above equations. For example, for perpendicular shocks with $n=5 \times 10^{13}$ per cm $\mathrm{cm}^{3}, a=100$, and $D$ $=200 \mathrm{~cm}$ (which implies $\beta=3.3$ ), one finds $T / B^{2}=0.0065$. The temperature, $T$, is determined by the shock velocity. In the simple model described above, the piston velocity determines the shock velocity. In an actual experiment, the shock velocity will be limited to what can be sustained throughout the experiment and is likely to decrease with time. On the one hand, for the experiment to make sense, the shocked temperature must be large compared to any ambient plasma temperature. On the other hand, the number of ions that can

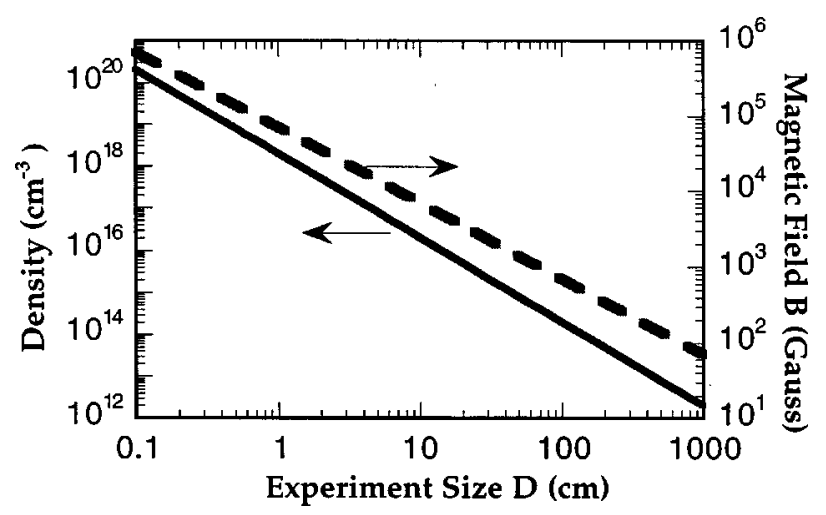

FIG. 4. Scaling of the density from holding $n D^{2}=2 \times 10^{18} \mathrm{~cm}^{-1}$. The magnetic-field scaling follows if one also requires $D / r_{\mathrm{Li}}=70$ and $T$ $=100 \mathrm{eV}$. be shocked and heated, with fixed energy, is more important than the temperature they reach. Beyond this, the shocked energy should be high enough to be easily measured. We will specify the experiment to achieve an initial shocked temperature of $100 \mathrm{eV}$. For fixed piston energy, the actual shocked temperature will be smaller by the time the shock reaches the end of the experiment. The shock velocity needed to heat hydrogen ions to $100 \mathrm{eV}$, using the standard relation $T=(3 / 16) M_{i} v_{s}^{2}$, is found to be $230 \mathrm{~km} / \mathrm{s}(2.3$ $\times 10^{7} \mathrm{~cm} / \mathrm{s}$ ). This requires a piston velocity of $173 \mathrm{~km} / \mathrm{s}$, which is also the post-shock flow velocity. The implications of this are discussed further below.

The design temperature of $100 \mathrm{eV}$ specifies the magnetic field by implication. First, for the perpendicular shock just discussed, this implies $B=120$ Gauss. We also note that the conditions just defined correspond, in the shocked plasma, to an ion cyclotron frequency, $\omega_{c i}=1.2 \times 10^{6} \mathrm{rad} / \mathrm{s}$, an ion gyroradius of $8.5 \mathrm{~cm}$, a shock transit time $\tau=9 \mu \mathrm{s}$, and $\omega_{c i} \tau$ $=40$. In the case of a parallel shock, the parameters $n=5$ $\times 10^{13} / \mathrm{cm}^{3}, a=70$, and $D=200 \mathrm{~cm}$ correspond to $\beta=6.4$ and $T / B^{2}=0.0008$. Thus, for $T=100 \mathrm{eV}$ one finds $B=350$ Gauss. Hence $\omega_{c i}=3.4 \times 10^{6} \mathrm{rad} / \mathrm{s}$, the ion gyroradius is $2.9 \mathrm{~cm}$, and $\omega_{c i} \tau \sim 30$. We will see below that the Alfvénic turbulence has ample time to grow under these conditions.

These results can be scaled to different conditions, using the above equations. For example, if one holds $D / r_{\mathrm{Li}}=70$, $T=100 \mathrm{eV}$, and $n D^{2}=2 \times 10^{18} \mathrm{~cm}^{-1}$, one obtains $\beta=6.4$, $M_{\mathrm{A}}=2.0$, and the scaling of $n$ and $B$ with $D$ shown in Fig. 4 . One sees that the required field strength grows rapidly as experiment size decreases.

\section{COLLISIONALITY}

The goal of the experiment is to create reproducible, well-diagnosed, relevant collisionless shocks. This statement, however, is insufficient to define the collisionality constraints on the design. One must ask just why one wants the system to be collisionless. There are at least four reasons. We survey them first, then discuss them in more detail. First, one needs to assure that the shock is not affected by collisions. This requires that the ion-ion collision mean free path be large compared to the shocked-ion gyroradius, $r_{\mathrm{Li}}$, which sets the scale of the shock transition. Second, one needs to assure that the collisions with electrons, whose principal effect is to slow down the ions by electron drag, do not cool the ions too much. The requirement must be that the corresponding cooling of the ions is a very small fraction of their shocked temperature. Third, the resistivity due to all collisions must be small enough that the MHD turbulence is not significantly affected. This is equivalent to requiring that the magnetic Reynolds number be large. Fourth, the collisions of ions with neutrals must be infrequent enough that charge exchange and other collisional processes are negligible. Now we take up these four issues in turn.

In evaluating ion-ion collisions, we consider two cases. First, we assess the corresponding mean free path of the shocked ambient ions. (The mean free path of any accelerated ions is longer.) The various (slowing, perpendicular scattering, and parallel) scattering rates are all comparable, 


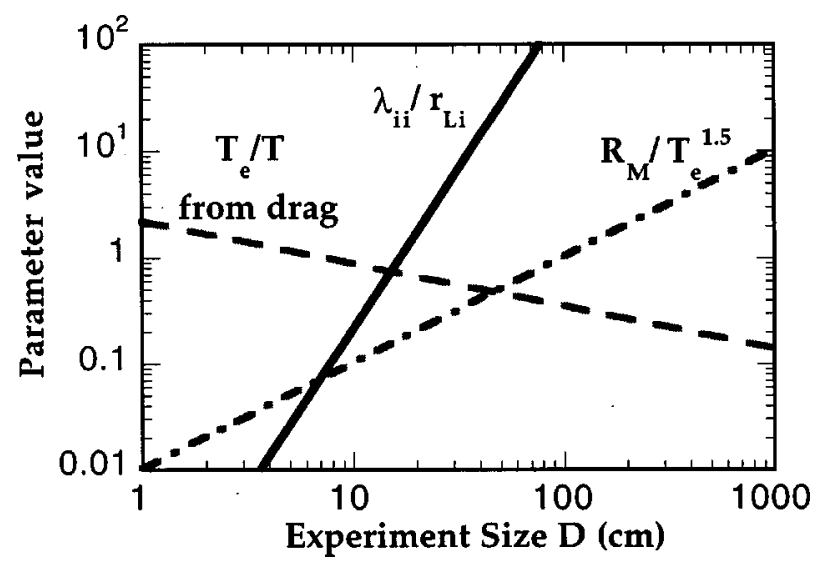

FIG. 5. Collisionality scaling parameters for the same conditions as Fig. 4 $\left(T=100 \mathrm{eV}, a=70\right.$, and $\left.n D^{2}=2 \times 10^{18} \mathrm{~cm}^{-1}\right)$.

of order $3 \times 10^{-7} n T^{3 / 2} \mathrm{~s}^{-1}$ (for a Coulomb logarithm of about 10). The corresponding mean free path, for hydrogen ions moving at $\sqrt{T / M_{i}}$, is $3 \times 10^{12} T^{2} / n \mathrm{~cm}$. For the nominal parameters given above, this is larger than $600 \mathrm{~cm}$, which compares to a gyroradius of about $3 \mathrm{~cm}$. The scattering of the fast, shock-reflected ions in the cool, unshocked ambient plasma is much smaller. The mean free path is $\sim 10^{16}\left(v_{s} / 100 \mathrm{~km} / \mathrm{s}\right)^{4} / n$. This, for the nominal parameters, is about $6000 \mathrm{~cm}$. Thus, the ion-ion scattering length, $\lambda_{i i}$, can be large compared to the ion gyroradius. The ratio can be expressed as

$$
\lambda_{i i} / r_{\mathrm{Li}}=3 \times 10^{12} T^{2} \sqrt{n D^{2}} /\left(n^{3 / 2} a\right) .
$$

Equation (6) is plotted in Fig. 5, along with other quantities discussed below. One can see that experiments following this scaling become collisional at sizes smaller than a few times $10 \mathrm{~cm}$. One could attempt to make the density lower, to reduce collisions, but only by sacrificing $\beta$ and/or $D / r_{\mathrm{Li}}$, according to Eqs. (1)-(5).

The second constraint is that the electrons should not heat to a large fraction of the ion temperature due to collisions. (The electrons also must not be too cold, as discussed next.) The electrons heat by drag on the ions. The drag rate decreases very strongly with increasing electron temperature, $T_{e}$, being proportional to $1 / T_{e}^{3 / 2}$. Thus, the electrons will heat until the heating rate drops to of the order of the experiment time. To evaluate this we integrate the electron heating equation, for cold electrons, $d T_{e} / d t=T \nu_{e i}$, where $\nu_{e i}=3.3$ $\times 10^{-8} n T_{e}^{-3 / 2} \mathrm{~s}^{-1}$ is the electron drag rate. The resulting temperature at time $\tau=D / v_{s}$ is

$$
T_{e}=\left(\frac{5 n T D}{6 \times 10^{7} v_{s}}\right)^{2 / 5} \mathrm{eV},
$$

which is about $27 \mathrm{eV}$ for the nominal parameters above. With some additional algebra, one can show that the scaling of $T_{e} / T$ is

$$
\frac{T_{e}}{T}=\left(\frac{2.3 \beta a^{2}}{D T^{2}}\right)^{2 / 5}=\left(\frac{3.7 \times 10^{-14} \sqrt{n} \sqrt{n D^{2}}}{T^{2}}\right)^{2 / 5} .
$$

This relationship is also shown in Fig. 5. One can see that, as $D$ drops increasingly below about $100 \mathrm{~cm}$, the electron heat- ing by drag becomes strong enough that the above model is not valid and that the ions will be strongly cooled by the electrons.

This takes us to the third constraint. Magnetic viscosity must not prevent or greatly alter the development of the MHD turbulence in the shock. We assess this by evaluating the magnetic Reynolds number. The magnetic Reynolds number, $R_{M}$ is given by

$$
R_{M}=\frac{L u}{D_{F}},
$$

where $L$ is a characteristic length scale, $u$ is the fluid velocity, and $D_{F}$ is the (kinematic) magnetic diffusivity. Since structures as small as $r_{\mathrm{Li}}$ may be important, we take $L \sim r_{\mathrm{Li}}$ $=D / a$. We take the (post-shock) flow velocity to be $u$ $\sim 1.8 \times 10^{7} \mathrm{~cm} / \mathrm{s}$, and

$$
D_{F}=2.5 \times 10^{7} \frac{Z}{T_{e}^{3 / 2}} \mathrm{~cm}^{2} / \mathrm{s},
$$

in which the ionic charge $Z=1$ for hydrogen.

Combining these equations, one finds $R_{M}=2.1 T_{e}^{3 / 2}$ for $D=200 \mathrm{~cm}$ and $a=70$, giving $R_{M} \sim 60$ for $T_{e} \sim 10 \mathrm{eV}$. The more general scaling relation is

$$
\frac{R_{M}}{T_{e}^{3 / 2}}=\frac{\sqrt{n D^{2}}}{a \sqrt{n}} \frac{u}{2.5 \times 10^{7}} .
$$

This relation is also shown in Fig. 5. For $T_{e} \sim 10 \mathrm{eV}$, for example, $R_{M}$ is about 30 times the value shown in the figure. We find that increasing $T_{e}$ is helpful because it increases the $R_{M}$. Paradoxically, the electron drag discussed above offers only limited help here, because it is too slow and occurs primarily downstream of the shock. It takes a substantial fraction of the experiment time for $T_{e}$ to increase by drag to the limit shown in Fig. 5. There are, however, other sources of electron heating, which can be sufficient to assure a large $R_{M}$.

There are at least three mechanisms that provide rapid electron heating. First, the plasma production method is likely to heat the electrons. Second, when the shock is not quasi-parallel, it causes the magnetic field to compress by a factor of 2-4. This will also heat the electrons. Third, there is believed to be some degree of electron heating associated with the MHD turbulence, although how much is unclear. Astrophysical measurements ${ }^{32}$ tend to suggest that $T_{e} / T$ may be 0.1 . Any effect that increases $T_{e}$ will increase $R_{M}$. There will also be some heating by drag on the ions reflected from the shock. By observing what actually happens, an experiment will also explore the dynamics of electron heating in collisionless shocks.

The fourth and final constraint (ion-neutral collisions) imposes conditions on the plasma production process. Charge exchange is the most important such process, and this must be small enough that many of the shock reflected ions reach the end of the experiment. The neutral particle density does not scale inherently with the design variables the way the other collisionality parameters do, but instead depends on how the plasma is produced. In general, however, this is a constraint that becomes more demanding as the 
density decreases. For example, the neutral $\mathrm{H}$ density could be as large as $3 \times 10^{13} \mathrm{~cm}^{-3}$ to achieve a $10 \mathrm{~cm}$ ion-neutral mean-free-path, but must be no larger that $3 \times 10^{12} \mathrm{~cm}^{-3}$ to keep this mean-free-path above $100 \mathrm{~cm}$. For very large experiments, which are otherwise favored, controlling the neutral density will be a key issue. We will leave the specific treatment of given cases, however, to the detailed design of such experiments.

\section{MHD TURBULENCE AND PARTICLE ACCELERATION}

Here we discuss the development of MHD turbulence in the shock and the consequent particle acceleration. The important questions are whether the turbulence will have time to develop and whether significant particle acceleration would be expected. These are both necessary if one is to learn from an experiment about the dynamics of particle acceleration in cosmic shocks.

The establishment of MHD turbulence depends on a number of mechanisms. A perpendicular shock can establish itself quite quickly, since it depends primarily on the behavior of reflected ions within a gyroradius in front of the shock. In this geometry, the piston pushes the field forward at high velocity. This should be sufficient to establish a shock.

Oblique and parallel shocks take longer, as they require the development of some MHD instability in the foreshock region. One can use the firehose instability as a surrogate for the broader class of instabilities that may participate in the shock dynamics. The pressure along the field lines due to the ions expanding from the energetic-ion source, such as a laser target, is much larger than the pressure perpendicular to them. For the ions moving at what will become the shock speed, the firehose instability ${ }^{33}$ has a growth rate given by $\gamma_{\mathrm{fh}} \sim k v_{s}$. Here we take the wavenumber of interest to correspond to the inverse gyroradius of the shock-reflected ions, $k \sim \omega_{c i} / v_{s}$. Thus, $\gamma_{\mathrm{fh}} \sim \omega_{c i}$ and the number of firehose growth times is $\omega_{c i} \tau$, given above as $(\sqrt{3} / 4) D / r_{\mathrm{Li}}$. For cases with $D / r_{\mathrm{Li}} \sim 50$ to 100 , the turbulence will experience a large number of growth times for the MHD instabilities that participate in collisionless shocks.

We thus conclude that collisionless shocks should develop in such a system. The next question, whether one will observe particle acceleration, has two parts. First, sufficiently energetic particles must be available for acceleration. Second, there must be a sufficient number of diffusive cycle times to allow substantial acceleration to occur. We address these in turn.

First, we require that particles be available for acceleration. This is known as the "injection" problem. Injection is not well understood. It has on the one hand been argued that the injection of enough particles into supernova shocks to account for the observed cosmic ray production is problematic. On the other hand, it has been argued that this conclusion, based on a test-particle analysis, is not valid ${ }^{34}$ and that injection is self-regulating. ${ }^{34,35}$ Experiments would help to reveal the injection dynamics. There are several reasons to believe that particles will be available for acceleration, and there are experimental options in addition. In the case of the parallel shock, the turbulence that establishes the shock grows within an injected population of high-velocity ions. Thus, ions will be available for acceleration from the start. Also, the shocked plasma is high-beta, which implies that the shocked thermal ions move as quickly as the Alfvénic turbulence. The perpendicular shock, in contrast, must produce its own ions for injection. As a result, one might see a significant difference in the particle distributions for parallel and perpendicular shocks. Nonetheless, this is a strong shock, which implies that the post-shock thermal velocity is about half the shock speed, meaning that a significant number of particles can cross the shock. Moreover, one could make special arrangements to provide an initial burst of very energetic ions if this were needed.

We next ask whether the particle distribution function will have time to evolve significantly during the experiment. Diffusive particle acceleration in shocks has been analyzed ${ }^{36}$ in terms of the characteristic time for a diffusive cycle, $T_{\mathrm{cyc}}$, given by

$$
T_{\mathrm{cyc}}=\left(\frac{\lambda_{1}}{u_{1}}+\frac{\lambda_{2}}{u_{2}}\right),
$$

where $\lambda$ is the mean-free-path, $u$ is the fluid velocity in the shock frame, and 1 and 2 designate the upstream and downstream sides of the shock, respectively. The mean-free-path is determined by the structure of the turbulence, and is at most of the order of the gyroradius of the shock reflected ions, $u_{1} / \omega_{c i}$. (This is a conservative estimate. It could also be of order the gyroradius of the shocked ambient ions, which would correspond to a smaller cycle time and to more particle acceleration.) Hence, one has $T_{\text {cyc }} \sim 5 / \omega_{c i}$ because $u_{2}=u_{1} / 4$, so that the number of diffusive cycles is $\tau / T_{\text {cyc }}$ $=(\sqrt{3} / 20)\left(D / r_{\mathrm{Li}}\right)$. In this nonrelativistic limit, the velocity increases by roughly $u_{1}$ per cycle. The upper limit on the acceleration is the energy at which the ion gyroradius becomes comparable to the system size. For a lateral size of $\sim 1 \mathrm{~m}$ in a $100 \mathrm{G}$ field this is $\sim 10 \mathrm{keV}$. If $u_{1}$ were $\sim 140$ $\mathrm{km} / \mathrm{s}$, this would require $\sim 10$ diffusive cycles, or $D / r_{\mathrm{Li}}$ $\sim 100$. Thus, an experiment that meets the constraints discussed in Secs. III and IV is likely to observe significant MHD turbulence and particle acceleration.

\section{PLASMA ISOLATION AND PRODUCTION}

Thus far we have investigated the scaling of plasma parameters corresponding to well-magnetized, high- $\beta$, and collisionless plasma. These scalings are independent of the specific approach to such experiments. In contrast, the exact magnetic field configuration and exact details of plasma production are inherently intertwined with the experimental approach. There are, however, some important general points to be made about these issues, which is our purpose in this section.

We first discuss the magnetic field configuration. If such experiments are to achieve the goal of being relevant to space and astrophysical systems, then the magnetic field lines in the system must have two properties. They must 


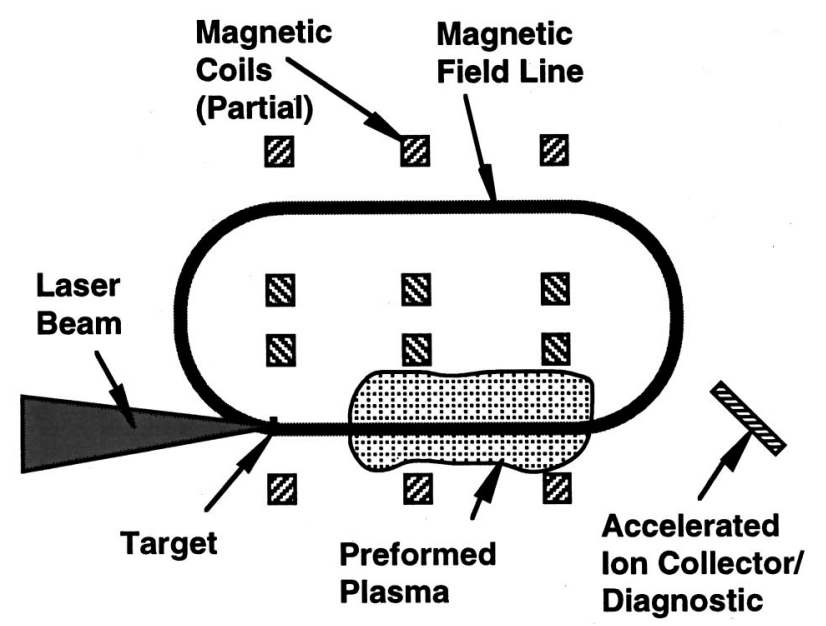

FIG. 6. Schematic of a possible experiment, using a laser to produce the piston. Some of the magnetic coils producing the racetrack field configuration are shown, but the coils that push the helical field lines into the page are not shown.

contact no material surfaces, and they must not loop around so as to connect the upstream side of the shock with the downstream side. We take these in turn.

Regarding the first property, the electron distribution function and the plasma potential are well known to be greatly altered when magnetic field lines contact a surface. Electron transport through the sheath at the surface alters the distribution, and conduction along the surface electrically connects the field lines. The electrons travel quite rapidly. Even at $10 \mathrm{eV}$, they move more than a meter per microsecond. Thus, the field topology should be designed to keep the field lines off of the walls. Consequently, they must be closed within the vacuum environment of the experiment. This means that at least some of the field coils will need to be enclosed within the vacuum environment.

Regarding the second property, it is not difficult to separate the upstream and downstream field lines for a perpendicular shock. For a parallel shock, however, this may be difficult. Reflected or accelerated ions and electrons stream out in front of a parallel shock. The field lines they flow along must not loop around to the downstream side of the shock but must also be kept off of the chamber walls. For the parallel shock, this may well require a helical field configuration in which such particles are shunted to one side (see Fig. 6). The accelerated ions can be allowed to escape the system, by taking advantage of their large orbits to collect them with surfaces or detectors where the field lines turn.

One potential advantage of collisionless shock experiments, not shared by magnetic confinement experiments, is that they need not be MHD stable. The shock wave described in previous sections will traverse the system in a small fraction of a typical growth time for a global MHD instability. (Local MHD turbulence will be driven to large amplitude, as is shown below.) From this point of view, one can use a manifestly unstable magnetic field configuration. In any given case, however, the need to produce the plasma in advance may impose some instability and confinement constraints.
There are a numerous options for plasma production. Some methods would involve causing the plasma to flow in along field lines. The Russian experiments, ${ }^{23,24}$ mentioned above, used a theta pinch in this way. One could use other sources as well, such as a Helicon source. In such cases, the best possible experiment would use fast field coils to separate the plasma of interest from the source before the shock was launched. Other methods would create the plasma in place. Given enough laser energy, one might photoionize the plasma by producing extreme ultraviolet emission from a laser-heated surface. Alternatively, using this or some other source of plasma initiation, one could employ rf heating. In these cases, one might use a supersonic gas valve as a source, so the neutral particles could drift out of the plasma before one launched the shock. Compared to most of the historical work, one has an advantage in producing plasma for these experiments, because it is not important to obtain a hot plasma and global MHD instabilities are not an issue, as long as they are slow enough. We leave further details of this topic to specific experiment designs.

\section{THE PISTON}

The next question is how to drive, through the medium just described, a shock having $v_{s}$ approaching $230 \mathrm{~km} / \mathrm{s}$. As was discussed in Sec. II, two of the approaches identified to date are the use of a theta pinch $^{14}$ and the use of a laser driver. ${ }^{24}$ Other methods might also be invented. Here we will discuss some of the issues associated with using a laser driver. Figure 6 shows a schematic of such an experiment, intended to study parallel shocks.

In all applications of a finite source of matter and energy to the problem of generating a collisionless shock, the finite mass of the source comes into play. The transition to a Sedov-Taylor expansion ${ }^{37,38}$ begins when the mass of the swept-up, ambient plasma equals that of the source. After this transition, the velocity of the blast wave decreases as time to the 0.6 power. In consequence, an experiment that generates a directed, slowly diverging plasma expansion can drive a shock for a much longer distance than an experiment producing a spherical expansion can.

There are at least three ways to use a laser driver to produce an expanding plasma. First, one can irradiate a small mass of material at comparatively high intensity, causing it to explode in a spherically expanding cloud of material. The main disadvantage of this method is that the expansion is spherical. Second, one can launch a shock through a block of matter, thick enough that the shock breaks out of the material after the laser pulse. In this case, the drifting matter and the expanding plasma on its leading edge can form a high Mach number plasma flow suitable for driving a shock. ${ }^{39,40}$ However, in order to achieve the necessary material velocities, a laser on the scale of the National Ignition Facility would be needed. Third, one can launch a shock through a block of matter thin enough that the shock breaks through the matter during the laser pulse, after which the entire block is accelerated further. This approach might be described as the "laser-driven rocket" approach, since the standard rocket 
equation describes the acceleration. This approach is preferable for experiments using a $\mathrm{kJ}$ class laser. We discuss it further next.

The theoretical hydrodynamic efficiency of a laserdriven rocket equals the fraction of the mass that is ablated from the target. Observations, and one-dimensional 1D hydrocode simulations, indicate that the actual value is about half of this. ${ }^{41}$ This leads one to use a thin target, so that a large fraction of the mass is ablated and the target is accelerated to large velocities. However, the accelerated target is subject to the Rayleigh Taylor instability and too thin a target will break up before the end of the laser pulse. An independent constraint is that the shock driven through the target by the laser ablation must be strong enough to ionize the entire target. This is necessary so that it becomes an expanding plasma as opposed to a cluster of fluid blobs.

One example of plausible parameters is as follows. A 1 $\mathrm{kJ}, 1 \mathrm{~ns}$ laser beam, at a wavelength of $0.53 \mu \mathrm{m}$, could irradiate a $1 \mathrm{~mm}$ diameter spot on an $18-\mu \mathrm{m}$ thick $\mathrm{CH}_{2}$ target. The corresponding average laser intensity is 1.3 $\times 10^{14} \mathrm{~W} / \mathrm{cm}^{2}$. The pressure generated is $12 \mathrm{Mbar}$ (scaling with laser intensity to the $2 / 3$ power). ${ }^{42}$ The pressure $p$ is related to the shock velocity $u_{s}$ by $p=2 \rho u_{s}^{2} /(\gamma+1)$, where $\rho$ is the initial density of the target $\left(\sim 1 \mathrm{~g} / \mathrm{cm}^{3}\right)$, from which $u_{s}=40 \mathrm{~km} / \mathrm{s}$. The sound speed in the shocked matter, $c_{0}$, is $22 \mathrm{~km} / \mathrm{s}$. The energy deposited in the target by shock heating is $6 \mathrm{~J}$. The actual hydrodynamic efficiency is $8.5 \%$, implying that the target acquires $85 \mathrm{~J}$ of kinetic energy, and is accelerated to about $120 \mathrm{~km} / \mathrm{s}$. According to simulations of this case, the post-shock acceleration is large enough that the plasma expansion from the rear surface is very limited until after the laser pulse.

The initial shock velocity will be much larger than 120 $\mathrm{km} / \mathrm{s}$. The leading edge of the target, which expands and accelerates, drives this shock through ambient plasma density that is about 10 orders of magnitude smaller than the target density. In the pure hydrodynamic case, the leading edge would accelerate to in excess of $200 \mathrm{~km} / \mathrm{s}$. In the actual plasma case, species separation and heat conduction can lead to larger final velocities. However, the shock would slow as the swept up mass approached the initial target mass. An actual experiment would vary the target properties in order to obtain an optimum forward shock strength and duration.

\section{CONCLUSION}

This concludes our examination of the problem of producing laboratory collisionless shocks of cosmic relevance. We identified nine constraints that must be met by such an experiment. We found that the key parameter that determines the degree to which one can meet the first three constraintslarge magnetization $\left(D / r_{\mathrm{Li}}\right)$, plasma beta $>1$, and Alfvén Mach number $>1$-is the product of density times the square of the experiment size, $n D^{2}$. Density is limited by the requirement that collisionality be small (the fourth constraint), with the implication that an experiment must be larger than some fraction of a meter in size. The ultimate size of such an experiment is limited by the size of feasible facilities and by the energy available to drive a shock through them. One does find, however, that an experiment that meets the first four constraints also will produce an adequate number of growth times for MHD turbulence to establish a collisionless shock, even in the parallel shock case, and an adequate number of diffusive cycles to observe substantial particle acceleration. Accomplishing this (meeting our eighth constraint) is the payoff from the point of view of being able to study mechanisms of relevance in cosmic shocks.

The other four constraints, pertaining to the production of the plasma, its isolation from material walls, driving the shock, and diagnosing the experiment, are much more specific to individual experimental designs. With one partial exception, we have left the treatment of these issues to attempts to design collisionless-shock experiments. The exception is the problem of using a laser to produce the "piston" that can drive a collisionless shock. We did see, by an example, that a kilojoule class laser could accomplish this goal.

It is the opinion of the author that experiments of this type are now feasible and should be undertaken. The funding of plasma physics has recently placed increasing importance on fundamental issues and sound basic research. One might hope that this will enable the resurgence of laboratory studies of collisionless shocks. The present paper should be of use to those who attempt to accomplish this.

\section{ACKNOWLEDGMENTS}

The author acknowledges the refinement of these ideas through numerous discussions of this topic with members of the laboratory, space, and astrophysical science communities. A partial list of these individuals includes Dmitri Ryutov, Bruce Remington, Robert Rosner, David Arnett, Tom Jones, Dan Winske, David Young, Tamas Gombosi, Len Fisk, and Nathan Schwadron.

This work was supported in part by the U.S. Department of Energy.

${ }^{1}$ R. Z. Sagdeev, Rev. Plasma Phys. 4, 23 (1966).

${ }^{2}$ N. F. Ness, C. S. Searce, and J. B. Seek, J. Geophys. Res. 69, 3531 (1964).

${ }^{3}$ A. R. Bell, Mon. Not. R. Astron. Soc. 182, 147 (1978).

${ }^{4}$ R. D. Blandford and J. P. Ostriker, Astrophys. J. Lett. 221, L29 (1978).

${ }^{5}$ W. I. Axford, E. Leer, and J. F. McKenzie, Astron. Astrophys. 111, 317 (1982).

${ }^{6}$ G. F. Krymsky, Dokl. Akad. Nauk SSSR 234, 1306 (1977).

${ }^{7}$ M. A. Lee, Rev. Geophys. Space Phys. 21, 324 (1983).

${ }^{8}$ M. A. Lee and L. A. Fisk, Space Sci. Rev. 32, 205 (1982).

${ }^{9}$ L. A. Fisk, J. Geophys. Res. 76, 1662 (1972).

${ }^{10}$ L. A. Fisk, J. Geophys. Res. 81, 4641 (1976).

${ }^{11}$ G. S. Podgorny and R. Z. Sagdeev, Sov. Phys. Usp. 12, 445 (1970).

${ }^{12}$ C. F. Kennel, J. P. Edmiston, and T. Hada, in Collisionless Shocks in the Heliosphere: A Tutorial Review, edited by R. G. Stone and B. T. Tsurutani (American Geophysical Union, Washington, DC, 1885), Vol. 34.

${ }^{13}$ D. Winske, M. E. Jones, A. G. Sgro, and V. A. Thomas (private communication), based on a workshop presentation.

${ }^{14}$ D. Biskamp, Nucl. Fusion 13, 719 (1973).

${ }^{15}$ E. Hintz, in Methods of Experimental Physics: Plasma Physics, edited by H. R. Griem and R. H. Lovberg (Academic, New York, 1970), Vol. 9A.

${ }^{16}$ P. E. Phillips and A. E. Robson, Phys. Rev. Lett. 29, 154 (1972).

${ }^{17}$ A. Y. Cheung, R. R. Goforth, and D. W. Koopman, Phys. Rev. Lett. 31, 429 (1973).

${ }^{18}$ R. P. Drake, J. Geophys. Res. 104, 14505 (1999).

${ }^{19}$ J. E. Borovsky, M. B. Pongatz, R. A. Roussel-Dupre, and T. H. Tan, Astrophys. J. 280, 802 (1984).

${ }^{20}$ J. Grun, J. Stamper, C. Manka, J. Resnick, R. Burris, J. Crawford, and B. H. Ripin, Phys. Rev. Lett. 66, 2738 (1991). 
${ }^{21}$ G. Dimonte and L. G. Wiley, Phys. Rev. Lett. 67, 1755 (1991).

${ }^{22}$ E. T. Vishniac, Astrophys. J. 274, 152 (1983).

${ }^{23}$ V. M. Antonov, V. P. Bashurin, A. I. Golubev, V. A. Ahmailo, Y. P. Zakharov, A. M. Orishich, A. G. Ponomarenko, V. G. Posukh, and V. N. Snytnikov, J. Appl. Mech. Tech. Phys. 26, 757 (1985).

${ }^{24}$ Y. P. Zakharov, A. M. Orishich, A. G. Ponomarenko, V. G. Posukh, and V. N. Snytnikov, "Laboratory simulation of collisionless coupling between Supernova remnants and magnetised interstellar medium,' Plasma Astrophysics: Proceedings of the Joint Varenna-Abastumani International School and Workshop (European Space Agency, Sukhumi, USSR, 1986), Vol. SP-251, p. 37.

${ }^{25}$ A. R. Bell, P. Choi, A. E. Dangor, O. Willi, D. A. Bassett, and C. J. Hooker, Phys. Rev. A 38, 1363 (1988).

${ }^{26}$ R. L. Berger, J. R. Albritton, C. Randall, C. Hanna, W. L. Kruer, A. B. Langdon, and E. A. Williams, Phys. Fluids B 3, 3 (1991).

${ }^{27}$ P. W. Rambo and J. Denavit, Phys. Plasmas 1, 4050 (1994).

${ }^{28}$ P. W. Rambo and R. J. Procassini, Phys. Plasmas 2, 3130 (1995).

${ }^{29}$ B. H. Ripin, E. A. McLean, C. K. Manka, C. Pawley, J. A. Stamper, T. A. Peyser, A. N. Mostovych, J. Grun, A. B. Hassam, and J. Huba, Phys. Rev. Lett. 59, 2299 (1987).

${ }^{30}$ B. H. Ripin, J. D. Huba, E. A. McLean, C. K. Manka, T. A. Peyser, H. R. Burris, and J. Grun, Phys. Fluids B 5, 3491 (1993).

${ }^{31}$ D. D. Ryutov, R. P. Drake, J. Kane, E. Liang, B. A. Remington, and M. Wood-Vasey, Astrophys. J. 518, 821 (1999).
${ }^{32}$ J. M. Laming, J. C. Raymond, B. M. McLaughlin, and W. P. Blair, Astrophys. J. 472, 267 (1996).

${ }^{33}$ N. A. Krall and A. W. Trivelpiece, Principles of Plasma Physics (San Francisco Press, San Francisco, 1986).

${ }^{34}$ M. A. Malkov, P. H. Diamond, and H. J. Volk, Astrophys. J. Lett. 533, L171 (2000)

${ }^{35}$ R. Blandford and D. Eichler, Phys. Rep. 154, 1 (1987).

${ }^{36}$ P. O. Lagage and C. J. Cesarsky, Astron. Astrophys. 118, 223 (1983).

${ }^{37}$ L. I. Sedov, Similarity and Dimensional Methods in Mechanics (Academic, New York, 1959), Vol. 1.

${ }^{38}$ S. G. Taylor, Proc. R. Soc. London, Ser. A 201, 192 (1950).

${ }^{39}$ R. P. Drake, J. J. Carroll, K. Estabrook, S. G. Glendinning, B. A. Remington, and R. McCray, Astrophys. J. Lett. 500, L157 (1998).

${ }^{40}$ R. P. Drake, S. G. Glendinning, K. Estabrook, B. A. Remington, R. McCray, R. Wallace, L. J. Suter, T. B. Smith, R. London, and E. Liang, Phys. Rev. Lett. 81, 2068 (1998).

${ }^{41}$ B. H. Ripin, R. Decoste, S. P. Obenshain, S. E. Bodner, E. A. McLean, F. C. Young, R. H. Whitlock, C. M. Armstrong, J. Grun, J. A. Stamper, S. H. Gold, D. J. Nagel, R. H. Lehmberg, and J. M. McMahon, Phys. Fluids 23, 1012 (1980).

${ }^{42}$ J. D. Lindl, Inertial Confinement Fusion (Springer-Verlag, New York, 1998). 